\title{
Successful long-term maintenance of Mansonella perstans in an in vitro culture system
}

\author{
Abdel Jelil Njouendou ${ }^{1,2+}$, Manuel Ritter ${ }^{3^{*}+}$, Winston Patrick Chounna Ndongmo ${ }^{1,2}$, Chi Anizette Kien ${ }^{1}$, \\ Gandjui Tchamatchoua Victor Narcisse ${ }^{1,2}$, Fanny Fri Fombad ${ }^{1,2}$, Dizzle Bita Tayong ${ }^{1,2}$, Kenneth Pfarr ${ }^{3,4}$, \\ Laura E. Layland ${ }^{3,4}$, Achim Hoerauf ${ }^{3,4 \dagger}$ and Samuel Wanji ${ }^{1,2+}$
}

\begin{abstract}
Background: Approximately 114 million people are infected with Mansonella perstans in large proportions of Africa. In contrast to other filariae that infect humans, M. perstans-infected individuals show no distinct pathology or specific clinical picture, indicating a well-tuned adaptation to the host. In addition, since M. perstans adult worms reside in serous cavities which are difficult to access, research has been hindered and there is a paucity of knowledge about the biology of $M$. perstans, especially the development of the different life stages as well as $M$. perstans-driven immune responses. Thus in this study, an in vitro culture system was developed which allows an in-depth analysis of M. perstans.

Results: Culicoides species were caught in Ediki (Kumba), Southwest Region within Cameroon following a blood meal on a microfilaremic donor that had 1500 microfilariae/ml of peripheral blood and kept in captivity for 12 days at $23^{\circ} \mathrm{C}$. In a pilot experiment, 15 infective larvae were obtained from the midges and co-cultured with a confluent monolayer of monkey kidney epithelial cells (LLC-MK2) in DMEM medium supplemented with 10\% FBS for up to 77 days. The resulting survival rates of $33 \%$ revealed that the cell-conditioned medium was suitable for long-term maintenance of M. perstans worms. To confirm these preliminary observations, 249 infective larvae were cultured for 50 days and their development was monitored daily and microscopically graded for motility. In total, 170 (68.3\%) filariae survived and 124 (49.8\%) larvae moulted between days 21-30 to become L5 stage larvae which were motile and showed continuous vigorous movement.
\end{abstract}

Conclusion: We have established an in vitro culture system for the generation and long-term maintenance of viable $M$. perstans worms. This technique will be an important tool to study parasite biology and development, the role in host immunity, and might be helpful to discover novel treatment strategies against this filariae.

Keywords: Mansonella perstans, In vitro culture, Worms, L3 infective larvae, Long-term maintenance

\section{Background}

Mansonella perstans is a well-adapted filarial nematode and, unlike in lymphatic filariasis (LF) or onchocerciasis, infection causes no distinct pathology with only mild symptoms like subcutaneous swellings, skin rashes and pleuritis $[1,2]$. It is estimated that 110 million people in over 33 countries, especially in the tropical parts of Latin America as well as large portions of Africa are infected

\footnotetext{
* Correspondence: manuel.ritter@ukb.uni-bonn.de

${ }^{\dagger}$ Equal contributors

${ }^{3}$ Institute of Medical Microbiology, Immunology and Parasitology, University

Hospital Bonn, Bonn, Germany

Full list of author information is available at the end of the article
}

$[1,2]$. The transmission of $M$. perstans requires a bite of a midge of the genus Culicoides [2], but in many endemic areas, the specific $M$. perstans-transmitting Culicoides species remains unclear. Upon transmission, the infective larvae (L3) develop into adult worms in an unknown manner and reside in serous body cavities [1-3]. Since $M$. perstans worms have only been recovered on rare occasions, research about the development of the different life stages remains limited. However, fecund female worms can release numerous microfilariae (Mf), which circulate in the peripheral blood and can be taken up by another Culicoides midge during a blood meal [1-3]. 
Although $M$. perstans Mf circulate during day and night in the peripheral blood [4], weak diurnal periodicity with a maximum Mf intensity during the first $12 \mathrm{~h}$ of the day has been observed [5]. In summary, many facets of $M$. perstans infection such as the development of life stages and transmission are still unclear. Thus being able to culture $M$. perstans in vitro would provide an essential tool in unravelling many of the open questions about this filarial nematode.

Several studies have reported in vitro cultures of Wuchereria bancrofti [6-8], Onchocerca [9-12] and Brugia species [13-15] that have also been used to test potential anti-filarial treatment strategies $[11,16,17]$. In this study, we have established an in vitro culture system to generate M. perstans worms from infective larvae. Overall, 249 infective larvae, obtained from 16 different collection batches of Culicoides, were co-cultured with monkey kidney epithelial cells (LLC-MK2) in DMEM medium supplemented with $10 \%$ FBS. High larvae survival rates of $68.3 \%$ revealed that the applied co-culture system was suitable for long-term maintenance of $M$. perstans for up to 50 days. Moreover, $49.8 \%$ of the larvae $(n=124)$ moulted between days $21-30$ of culturing to become L5 stage larvae which were vigorously motile.

\section{Methods}

\section{Isolation of infective $M$. perstans larvae}

Mansonella perstans infective larvae (L3) were obtained from Culicoides midges following a blood meal on a consenting donor from Ediki village (Kumba health district) that had a peripheral microfilariae (Mf) load of $1500 \mathrm{Mf} / \mathrm{ml}$. After 15-20 min, midges were collected via aspiration into a net in darkness. Midges were then aspirated using bright torch light into tubes. This process was repeated several times between $6 \mathrm{pm}$ and $6 \mathrm{am}$. Engorged midges were kept in $50 \mathrm{ml}$ tubes filed onefourth with plaster of Paris which formed a cement layer and helped to retain moisture for 12 days at $23{ }^{\circ} \mathrm{C}$, the time required for the development of L3 from $\mathrm{Mf}$ in the vector. Midges were fed daily with cotton gauze soaked in autoclaved $15 \%$ sucrose solution suspended over the rim of moist paper. At intervals of $24 \mathrm{~h}$, a drop of distilled water was added to the plaster of Paris to keep the gauze moist. After 12 days, L3 were isolated in dissecting medium containing RPMI-1640 medium (Sigma-Aldrich, Munich, Germany) supplemented with a $2 \%$ antibiotic cocktail (pencillin-streptomycin-neomycin; Thermo Fisher Scientific, Schwerte, Germany) using a dissecting microscope (Motic, Wetzlar, Germany). The head, the thorax and the abdomen were separately placed in three different dissecting wells containing the same dissecting medium. Infective larvae were allowed to migrate out of the various parts, especially the proboscis. The infective larvae were collected in sterile manner and parasites were washed 4 times with sterile dissecting medium and used to culture M. perstans worms in vitro.

\section{In vitro culturing of $M$. perstans worms}

In 48-well flat bottomed plates (Greiner Bio-One $\mathrm{GmbH}$, Frickenhausen, Germany), $1 \mathrm{ml}$ of Dulbecco's Modified Eagle Medium (DMEM; Thermo Fisher Scientific, Schwerte, Germany) supplemented with $10 \%$ foetal bovine serum (FBS; Lonza, Verviers, Belgium), $5 \mu \mathrm{g} / \mathrm{ml}$ ciprofloxacin hydrochloride and $10 \mu \mathrm{g} / \mathrm{ml}$ fluconazole or $1 \%$ penicillin-streptomycin-neomycin (PSN) (Sigma-Aldrich) was used for the in vitro culture of M. perstans. Ten L3 were cultured in a 48-well plate (Thermo Fisher Scientific) containing a confluent monolayer of monkey kidney epithelial cells (LLC-MK2; LGC Standard GmbH, Wesel, Germany) which were cultivated 2 days before the addition of L3. Four to ten infective larvae (average of 6-7 larvae/ well) were cultured for at least 50 days at $37^{\circ} \mathrm{C}$ and $5 \%$ $\mathrm{CO}_{2}$ and were monitored daily using a Motic AE21 inverted microscope (Motic, Wetzlar, Germany). Additionally, growth of the worms was recorded using a digital camera (Fiber Tech PC \& Mobile, Putsebocht, Netherlands). Helminth viability was assessed on a daily basis via microscopy using a 4 point score sheet: 0 , no movement; 1 , intermittent shaking of head and tail; 2, sluggishness (movement of whole worm on one spot); and 3, vigorous movement. Mortality was defined as the percentage of dead worms in each well. An immotile worm was declared dead after observing its immobility for $5 \mathrm{~min}$. Moulting and motility were scored for individual worms by two experienced trained parasitologists and results were analysed as an aggregate. The culture medium was replenished at 5 day intervals or as soon as observable colour changes were seen in the wells.

\section{Data processing and analysis}

Data were collected daily on record sheets and entered into a template designed on Microsoft Office Excel 2007 (Microsoft, Redmond, USA). Graphs were created using PRISM 5 for Windows (GraphPad Software, Inc., La Jolla, USA).

\section{Results}

Establishment of a cell-conditioned in vitro culture system for the maintenance of $M$. perstans worms

Due to the inaccessibility of adult $M$. perstans worms, investigations into the developmental stages and metamorphosis of infective larvae into adult worms have not been possible until now. Therefore, we developed an in vitro culture to generate viable $M$. perstans worms from infective larvae (L3). Several studies previously described that cell-conditioned media, especially cocultures with monkey kidney epithelial cells and inactivated FBS, were critical components for the maintenance of 
filariae in vitro [8-13]. Based on those studies, we established cultures of monkey-kidney epithelial cells in DMEM medium supplemented with $10 \%$ foetal bovine serum and successfully cultured Loa loa in vitro (unpublished findings). Based on these observations, we hypothesised that this culturing method could also be suitable for the development and growth of $M$. perstans infective larvae. Indeed, long-term maintenance of infective $M$. perstans larvae was observed under these culture condition with $40 \%(n=6)$ worm survival after 31 days and $33 \%(n=5)$ surviving for up to 77 days in the in vitro culture (Fig. 1).

\section{Development of viable $M$. perstans worms from infective larvae (L3) in vitro}

Since we were able to maintain worms in co-culture with LLC-MK2 cells, we used the co-culture to analyse the metamorphosis and motility of L3 in more detail. Thus, 249 infective larvae were extracted from 16 Culicoides batches following a blood meal on an $M$. perstans $\mathrm{Mf}+$ individual and cultured as described in the Methods section. During the 50 day culturing process, the viability and activity of each nematode was assessed on a daily basis by visual observation until they died. The motility and survival of the developing worms was monitored using a 4 point scoring system as described above. All infective larvae recovered after dissection had a starting score of 2 (Fig. 2a). After 50 days of in vitro culture, $31.7 \%(n=79)$ of the worms were immotile (score 0 ), but overall, 68.3\% $(n=170)$ survived (score $1-3)$. In addition, the activity of the worms with score $3(49.8 \%, n=124)$ increased over the period of the in vitro culture, whereas the percentage $(13.7 \%)$ and number $(n=34)$ of worms

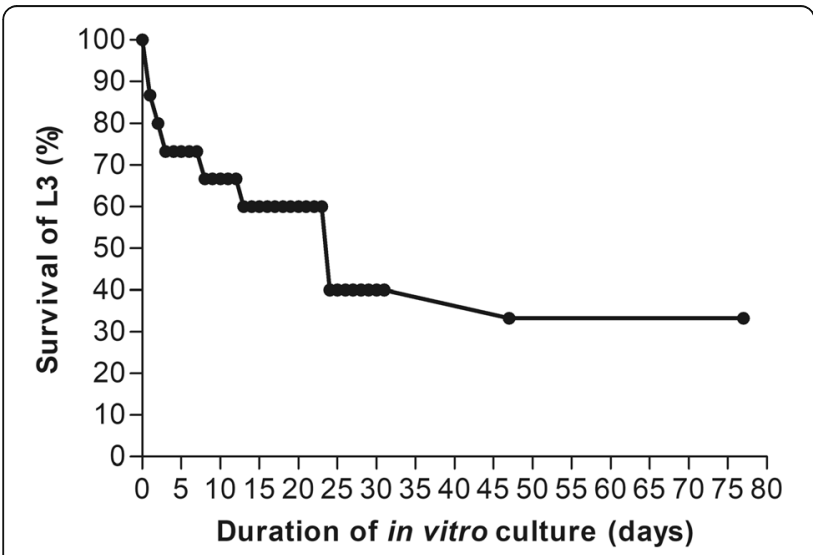

Fig. 1 In vitro long-term maintenance of $M$. perstans infective larvae cocultured with monkey kidney epithelia cells (LLC-MK2). M. perstans infective larvae ( $\mathrm{L} 3 ; n=15)$ were co-cultured with monkey kidney epithelia cells (LLC-MK2) in DMEM medium supplemented with 10\% FBS at $37{ }^{\circ} \mathrm{C}$ and $5 \% \mathrm{CO}_{2}$ for 77 days. Cultures were monitored microscopically to determine survival rates (\%) of $M$. perstans worms with sluggish movement (score 2) decreased. Interestingly, $60.2 \%(n=150)$ of the larvae moulted and became L5 stage larvae between days 21-30 ( $n=127$, Fig. 2b), which corresponded to the increase in viability observed through score 3. Furthermore, videos recorded on day 20 (Additional file 1), 30 (Additional file 2), 40 (Additional file 3) and 50 (Additional file 4) show the development and growth of $M$. perstans worms over the time of in vitro culture. To get an impression of the worm size, we randomly obtained five worms from the in vitro culture and measured the length. Interestingly, the worms had lengths of 40-60 mm $($ mean $=48.4 \mathrm{~mm}$, median $=47 \mathrm{~mm} ; \mathrm{SD}=8.2 \mathrm{~mm})$ after 50 days of in vitro culture.

\section{Discussion}

The silent nature of $M$. perstans with regards to clinical symptoms and pathology development has resulted in a shortfall on studies on the biology, transmission and host evasion tactics of this nematode. Interestingly $M$. perstans is, however, considered one of the most prevalent human diseases in tropical areas with approximately 114 million infected people $[1,2,18]$. Most of the knowledge about $M$. perstans infection has been obtained as a by-product of studies with other filarial or parasitic infections [19-22] that were considered to be more important for public health. However, M. perstans-induced symptoms such as subcutaneous swellings, abdominal pain, skin rashes, pericarditis and pleuritis $[1$, 2] have been reported. Moreover, initial studies have shown that $M$. perstans infection may influence the efficacy of Bacillus Calmette-Guérin (BCG) vaccination against tuberculosis as well as the susceptibility and disease course of HIV, tuberculosis and malaria [23-26]. Those studies increased the focus on $M$. perstans research but, until now, investigations have been hindered by the inaccessibility of $M$. perstans adult worms [1-3]. Therefore, in this study we established an in vitro culture system to decipher unresolved questions about the biology and development of $M$. perstans worms. To the best of our knowledge, we demonstrate for the first time that cultures of monkey kidney epithelial cells (LLC-MK2) in DMEM medium supplemented with $10 \%$ FBS allow long term-maintenance of $M$. perstans worms for up to 77 days. These data confirm previous studies showing that cell-conditioned and inactivated FBS are critical for the maintenance of filariae in vitro [8-13]. In addition, further experiments have shown that the in vitro survival and maintenance of Loa loa infective larvae was improved in DMEM medium compared to RPMI and that LLC-MK2 cells are indispensible for parasite moulting (unpublished findings). Collectively, these data suggest that Loa loa and M. perstans may 


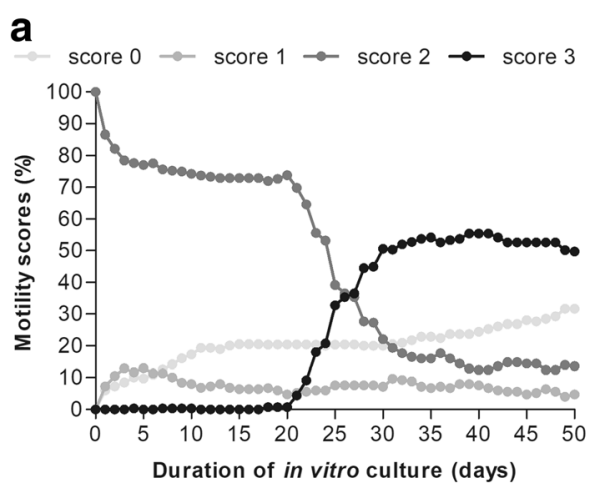

b

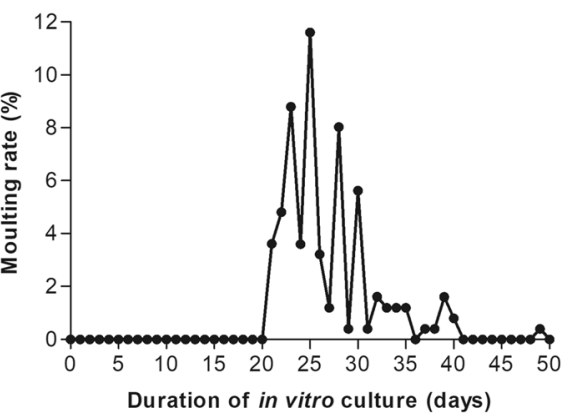

Fig. 2 Mansonella perstans infective larvae moult and developed into viable and motile worms in vitro. M. perstans infective larvae $(n=249)$ were cocultured with monkey kidney epithelia cells (LLC-MK2) in DMEM medium supplemented with $10 \% \mathrm{FBS}$ at $37{ }^{\circ} \mathrm{C}$ and $5 \% \mathrm{CO}_{2}$ for 50 days. The cultures were monitored on a daily basis via microscopy to determine (a) worm motility (\%) according to a 4 point scale [0, no movement; 1 , intermittent shaking of head and tail; 2 , sluggish (shaking of the whole worm on a spot); and 3, vigorous movement] and (b) moulting rates (\%) of the developing M. perstans worms

share similar in vitro requirements for growth and development. In addition, most of the $M$. perstans infective larvae moulted between days 21-30 to become L5 stage larvae which continued to show vigorous movement upon 50 days of in vitro culture. It is known that moulting behaviour and life duration of the different larval stages varies strongly between different filariae and indeed, there are two groups of filaria based on the duration of L3 stages in their mammalian host [27]. The first group including filariae such as Onchocerca volvulus and O. lienalis is characterized by an early moult (less than 3 days) whereas other filariae like L. loa, Wuchereria bancrofti and Brugia spp. execute the first moult only after a week. In this latter group, the second moult (L4 into L5) occurs significantly before day $50[27,28]$. Mansonella perstans therefore seem to belong to the first group which execute an early moult from L3 into L4 stage larvae within the first 3 days of in vitro culture. Thus, it is likely that the first moult remains unnoticed or was not obvious during this study and the moult observed after 20 days marks the development from L4 into L5 stage larvae. However, in-depth analysis needs to be performed to decipher $M$. perstans moulting behaviour and the development of the different life stages in vitro in greater detail. Interestingly, the worms had lengths of $40-60 \mathrm{~mm}$ after 50 days of in vitro culture and are thus comparable to M. perstans worms (35$80 \mathrm{~mm}$ ) isolated from infected human individuals [29]. Nevertheless, further investigations need to be performed to discriminate gender and decipher morphology and fertility of the cultured M. perstans worms in more detail. In addition, the in vitro culture system will allow us to elucidate the presence and role of Wolbachia in this filarial nematode as previous studies have reported contradictory findings showing either the absence $[30,31]$ or the presence of the endosymbiont in M. perstans worms [32-34].

\section{Conclusions}

Collectively, these findings present a novel tool for $M$. perstans research and an approach to elucidate several unresolved questions about the biology and development of $M$. perstans life stages. These include studies about worm fertility and morphology, genome and transcriptome analysis with single sex adult worms, as well as indepth analysis of the role of Wolbachia in M. perstans worms using immunohistochemistry, multilocus sequence typing, and treatment with anti-wolbachial antibiotics. Finally, the in vitro worm culture also provides a platform to study potential treatments against this ivermectin nonsusceptible filariae $[35,36]$.

\section{Additional files}

Additional file 1: Mansonella perstans worms after 20 days of in vitro culture. On day 20 of the in vitro culture, motility of $M$. perstans worms were microscopically monitored and recorded at 10x magnification. (MP4 $1737 \mathrm{~kb}$ )

Additional file 2: Mansonella perstans worms after 30 days of in vitro culture. On day 30 of the in vitro culture, motility of $M$. perstans worms were microscopically monitored and recorded at 10x magnification. (MP4 $2282 \mathrm{~kb}$ )

Additional file 3: Mansonella perstans worms after 40 days of in vitro culture. On day 40 of the in vitro culture, motility of $M$. perstans worms were microscopically monitored and recorded at 10x magnification. (MP4 4426 kb)

Additional file 4: Mansonella perstans worms after 50 days of in vitro culture. On day 50 of the in vitro culture, motility of M. perstans worms were microscopically monitored and recorded at $4 \times$ magnification. (MP4 $6039 \mathrm{~kb})$

\section{Abbreviations}

DMEM: Dulbecco's modified eagle medium; FBS: Foetal bolllvine serum; HIV: Human immunodeficiency virus; L3: Infective larvae (third stage larvae able to infect human hosts); Mf: Microfilariae

\section{Acknowledgements}

We sincerely thank the chiefs and entire populations of all health districts surveyed for accepting and participating in this study. The authors are grateful 
to the participants for their voluntary participation. We also thank the Cameroon health system for all the facilities put in place during the surveys.

\section{Funding}

This work is funded through a grant awarded to SW, LEL and AH from the German Research Council (DFG) within the "African-German Cooperation Projects in Infectiology" (HO 2009/10-1). AH and LEL are members of the Excellence Cluster Immunosensation (DFG, EXC 1023). AH, LEL and KP are members of the German Centre of Infectious Disease (DZIF).

\section{Availability of data and materials}

The datasets used and/or analysed during the current study are available from the corresponding author upon reasonable request.

\section{Authors' contributions}

SW and AH conceived and designed the study. AJN, FFF and WPCN developed the in vitro culture of $M$. perstans worms. WPCN, CAK, GTVN and DBT managed field studies. SW, AJN and MR analysed and interpreted data sets. MR wrote the manuscript which was then critically assessed and amended by SW, AH, LEL and KP. All authors read and approved the final manuscript.

\section{Ethics approval and consent to participate}

An ethical clearance was obtained from the National Institutional Review board, Yaoundé (REF: No. 2015/09/639/CE/CNERSH/SP) and administrative clearance from the Delegation of Public Health, South West Region (Re: R11/ MINSANTE/SWR/RDPH/PS/259/382). Special consideration was taken to minimize the health risks to which any participant in this study was exposed. We explained the objectives of the study to the donor willing to participate after which they signed an informed consent form. The participant's documents were given a code to protect the privacy of the study subject. At the end of the study, the donor received a cure of mebendazole (100 mg twice a day for 30 days) against the infection of $M$. perstans [2]

\section{Consent for publication}

Not applicable.

\section{Competing interests}

The authors declare that they have no competing interests.

\section{Publisher's Note}

Springer Nature remains neutral with regard to jurisdictional claims in published maps and institutional affiliations.

\section{Author details}

${ }^{1}$ Parasite and Vector Research Unit (PAVRU), Department of Microbiology and Parasitology, University of Buea, Buea, Cameroon. ${ }^{2}$ Research Foundation for Tropical Diseases and the Environment (REFOTDE), Buea, Cameroon. ${ }^{3}$ Institute of Medical Microbiology, Immunology and Parasitology, University Hospital Bonn, Bonn, Germany. ${ }^{4}$ German Centre for Infection Research (DZIF), Bonn-Cologne partner site, Bonn, Germany.

\section{Received: 16 August 2017 Accepted: 31 October 2017}

Published online: 10 November 2017

\section{References}

1. Hoerauf A. Mansonella perstans - the importance of an endosymbiont. N Engl J Med. 2009;361:1502-4

2. Simonsen PE, Onapa AW, Asio SM. Manosnella perstans filariasis in Africa. Acta Trop. 2011;120(Suppl 1):S109-20.

3. Asio SM, Simonsen PE, Onapa AW. Mansonella perstans filariasis in Uganda: patterns of microfilaraemia and clinical manifestations in two endemic communities. Trans R Soc Trop Med Hyg. 2009;103:266-73.

4. Kershaw WE. Studies on the epidemiology of filariasis in West Africa, with special reference to the British Cameroons and the Niger Delta. I. Methods of survey for infections with Loa loa and Acanthocheilonema perstans. Ann Trop Med Parasitol. 1950;44:361-78.

5. Asio SM, Simonsen PE, Onapa AW. Analysis of the $24 \mathrm{~h}$ microfilarial periodicity of Mansonella perstans. Parasitol Res. 2009;104:945-8.
6. Franke ED, Riberu W, Wiady I. In vitro cultivation of third stage larvae of Wuchereria bancrofti to the fourth stage. Am J Trop Med Hyg. 1987;37:370-5

7. Hoti SL, Gros AM, Paily KP, Manonmani AM, Mary KA, Balaraman K. In vitro cultivation of third stage larvae of Wuchereria bancrofti to fourth stage: influence of some physico-chemical factors. Southeast Asian J Trop Med Public Health. 1994;25:278-83.

8. Zaraspe G, Cross JH. Attempt to culture Wuchereria bancrofti in vitro. Southeast Asian J Trop Med Public Health. 1986;17:579-81.

9. Townson S, Connelly C, Muller R. Optimization of culture conditions for the maintenance of Onchocerca gutturosa adult worms in vitro. J Helminthol. 1986;60:323-30.

10. Townson S. The development of a laboratory model for onchocerciasis using Onchocerca gutturosa: in vitro culture, collagenase effects, drug studies and cryopreservation. Trop Med Parasitol. 1988;39(Suppl 4):475-9.

11. Townson S, Tagboto S, McGarry HF, Egerton GL, Taylor MJ. Onchocerca parasites and Wolbachia endosymbionts: evaluation of a spectrum of antibiotic types for activity against Onchocerca gutturosa in vitro. Filaria J. 2006;5:4.

12. Cupp MS, Cupp EW, Poulopoulou C. The use of cell-conditioned medium for the in vitro culture of Onchocerca spp. larvae (Nematoda: Filarioidea). Trop Med Parasitol. 1990;41:20-4.

13. Mak JW, Lim PK, Sim BK, Liew LM. Brugia malayi and B. pahangi: cultivation in vitro of infective larvae to the fourth and fifth stages. Exp Parasitol. 1983;55:243-8.

14. Riberu WA, Atmosoedjono S, Purnomo, Tirtokusumo S, Bangs MJ, Baird JK Cultivation of sexually mature Brugia malayi in vitro. Am J Trop Med Hyg. 1990;43:3-5

15. Falcone $\mathrm{FH}$, Zahner $\mathrm{H}$, Schlaak $\mathrm{M}$, Haas $\mathrm{H}$. In vitro cultivation of thirdstage larvae of Brugia malayi to the young adult stage. Trop Med Parasitol. 1995:46:230-4

16. Baird JK, Riberu W, Masbar S, Purnomo. Ivermectin inhibits molting of Wuchereria bancrofti third stage larvae in vitro. J Parasitol. 1991;77:162-3.

17. Townson S, Connelly C, Dobinson A, Muller R. Drug activity against Onchocerca gutturosa males in vitro: a model for chemotherapeutic research on onchocerciasis. J Helminthol. 1987;61:271-81.

18. Nelson GS. Filarial infections as zoonoses. J Helminthol. 1965;39:229-50.

19. Metenou S, Dembélé B, Konate S, Dolo H, Coulibaly SY, Coulibaly YI, et al. Patent filarial infection modulates malaria-specific type 1 cytokine responses in an IL-10-dependent manner in a filaria/malaria-coinfected population. J Immunol. 2009;183:916-24.

20. Semnani RT, Mahapatra L, Dembele B, Konate S, Metenou S, Dolo H, et al. Expanded numbers of circulating myeloid dendritic cells in patent human filarial infection reflect lower CCR1 expression. J Immunol. 2010;185:6364-72

21. Dolo H, Coulibaly YI, Dembele B, Konate S, Coulibaly SY, Doumbia SS, et al. Filariasis attenuates anemia and proinflammatory responses associated with clinical malaria: a matched prospective study in children and young adults. PLoS Negl Trop Dis. 2012;6:e1890.

22. Lechner CJ, Komander K, Hegewald J, Huang X, Gantin RG, Soboslay PT, et al. Cytokine and chemokine responses to helminth and protozoan parasites and to fungus and mite allergens in neonates, children, adults, and the elderly. Immun Ageing. 2013:10:29.

23. Muhangi L, Woodburn P, Omara M, Omoding N, Kizito D, Mpairwe H, et al. Associations between mild-to-moderate anaemia in pregnancy and helminth, malaria and HIV infection in Entebbe, Uganda. Trans R Soc Trop Med Hyg. 2007;101:899-907.

24. Hillier SD, Booth M, Muhangi L, Nkurunziza P, Khihembo M, Kakande M, et al. Plasmodium falciparum and helminth coinfection in a semi urban population of pregnant women in Uganda. J Infect Dis. 2008;198:920-7.

25. Stensgaard AS, Vounatsou P, Onapa AW, Utzinger J, Pedersen EM, Kristensen TK, et al. Ecological drivers of Mansonella perstans infection in Uganda and patterns of co-endemicity with lymphatic filariasis and malaria. PLoS Negl Trop Dis. 2016;10:e0004319.

26. Elliott AM, Mawa PA, Webb EL, Nampijja M, Lyadda N, Bukusuba J, et al. Effects of maternal and infant co-infections, and of maternal immunisation, on the infant response to BCG and tetanus immunisation. Vaccine. 2010:29:247-55.

27. Bain O, Wanji S, Enyong P, Petit G, Noireau F, Eberhard Ml, et al. New features on the moults and morphogenesis of the human filaria Loa loa by using rodent hosts consequences. Parasite. 1998;5:37-46. 
28. Bianco AE, Mustafa MB, Ham PJ. Fate of developing larvae of Onchocerca lienalis and $O$. volvulus in micropore chambers implanted into laboratory hosts. J Helminthol. 1989;63:218-26.

29. Baird JK, Neafie RC, Lanoie L, Connor DH. Adult Mansonella perstans in the abdominal cavity in nine Africans. Am J Trop Med Hyg. 1987;37:578-84.

30. Büttner DW, Wanji S, Bazzocchi C, Bain O, Fischer P. Obligatory symbiotic Wolbachia endobacteria are absent from Loa loa. Filaria J. 2003;2:10.

31. Grobusch MP, Kombila M, Autenrieth I, Mehlhorn H, Kremsner PG. No evidence of Wolbachia endosymbiosis with Loa loa and Mansonella perstans. Parasitol Res. 2003;90:405-8.

32. Keiser PB, Coulibaly Y, Kubofcik J, Diallo AA, Klion AD, Traoré SF, et al. Molecular identification of Wolbachia from the filarial nematode Mansonella perstans. Mol Biochem Parasitol. 2008;160:123-8.

33. Coulibaly YI, Dembele B, Diallo AA, Lipner EM, Doumbia SS, Coulibaly SY, et al. A randomized trial of doxycycline for Mansonella perstans infection. $\mathrm{N}$ Engl J Med. 2009;361:1448-58.

34. Gehringer C, Kreidenweiss A, Flamen A, Antony JS, Grobusch MP, Bélard S. Molecular evidence of Wolbachia endosymbiosis in Mansonella perstans in Gabon, Central Africa. J Infect Dis. 2014;210:1633-8.

35. Asio SM, Simonsen PE, Onapa AW. Mansonella perstans: safety and efficacy of ivermectin alone, albendazole alone and the two drugs in combination. Ann Trop Med Parasitol. 2009:103:31-7.

36. Asio SM, Simonsen PE, Onapa AWA. Randomised, double-blind field trial of ivermectin alone and in combination with albendazole for the treatment of Mansonella perstans infections in Uganda. Trans R Soc Trop Med Hyg. 2009;103:274-9.

\section{Submit your next manuscript to BioMed Central and we will help you at every step:}

- We accept pre-submission inquiries

- Our selector tool helps you to find the most relevant journal

- We provide round the clock customer support

- Convenient online submission

- Thorough peer review

- Inclusion in PubMed and all major indexing services

- Maximum visibility for your research

Submit your manuscript at www.biomedcentral.com/submit 